\title{
Development of Ultra-thin TEM Lamella Preparation Technique and Its Application in Failure Analysis
}

Yu Zhang, Brian Popielarski, Kevin Davidson, Long Men, Wayne Zhao and Frieder Baumann

GLOBALFOUNDRIES US Inc., Malta, New York, United States

The preparation of TEM lamellae plays a critical role in physical failure analysis of semiconductor devices [1]. As the size of device continues to shrink, in-depth understanding of more subtle defects becomes key for root cause analysis to facilitate line yield improvement. However, the size and complex nature of the defects pose great challenges to TEM lamella preparation and analysis. Projection effects related to lamella thickness could lead to misinterpretation of the defects. Therefore, preparation of ultra-thin TEM lamella has to be explored and will be one of the approaches that could address these challenges.

Traditional focused ion beam (FIB) TEM sample preparation generally consists of targeting, coating, bulk milling, lift-out and final thinning [2]. For normal upright sample preparation, $\sim 50 \mathrm{~nm}$ sample thickness can be achieved with $\sim 8 \mathrm{~nm}$ total Si damage on each side [3]. Thinner ( 20nm) sample with $\sim 1-2 \mathrm{~nm} \mathrm{Si}$ damage can be achieved using inverted thinning geometry and $2 \mathrm{KV}$ ion beam. However, ion-beam damage and resolution limit make the preparation of even thinner TEM lamella extremely challenging.

In our recent developments on ultra-thin TEM specimen preparation, a commercially available dual beam FIB tool was used. Thinning of the sample was performed inverted and included a sequence of glancing angles from $1^{\circ}$ to $8^{\circ}$. Final cleaning of the sample surfaces was performed at $500 \mathrm{~V}$ which allowed for clean surface and minimum surface amorphisation. The final TEM sample thickness varies from 10-15nm. These improvements allow us to prepare high quality, sub-20nm thick TEM lamellae targeting subtle defects which cause mainly soft (electrical) fails in advanced FinFET devices [4].

As an example, source to drain leakage was identified by electrical fault isolation at a failing transistor. The sample was first prepared thick. Crystal defects and undesired P/As signals were detected at fin tips as shown in Fig.1 and 2. It is not clear if the observed defects were located in the channel or source/drain areas due to possible projection effects. In order to further verify the defect and fully understand the root cause, two sequential re-thinning and re-imaging cycles were performed on the sample. All 3 sets of data were then compared to differentiate the real defect from any projection effects. Top row of Fig. 3 shows the X-cut view of the different locations and thicknesses of the TEM lamella. The gradually decreased thickness of the TEM lamella can be evidenced by the decreasing Hf signals from Y-cut sample in bottom row of Fig.3, indicating that the PC sidewalls were partially (Fig.3 (d)) or completely (Fig.3 (f)) removed. No crystal defects observed from the same area after re-thinning as shown in Fig.4 indicates the elimination of projection effect. Significant decrease of As and P signals at fin tips after re-thinning as shown in Fig.5 further confirmed that the previously observed anomalies came from projection effect.

In conclusion, ultra-thin TEM lamella could help eliminate projection effect and significantly improve understanding of the defects, especially subtle defects in most advanced semiconductor devices. 

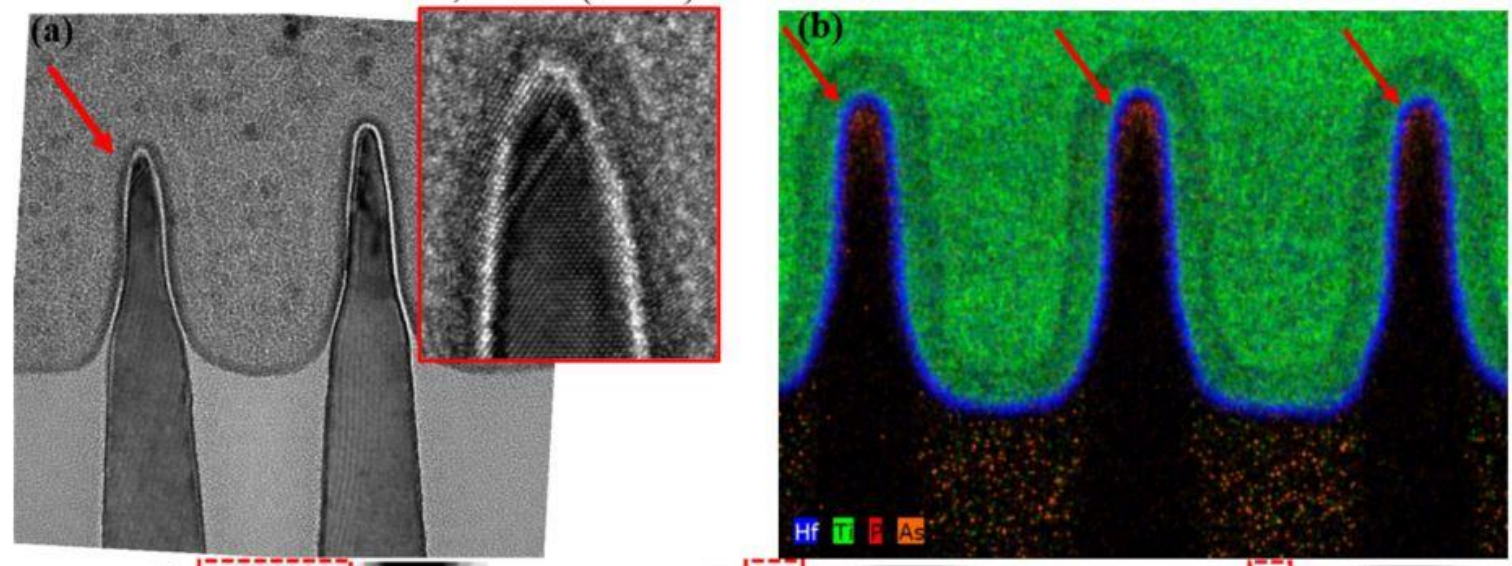

(c)

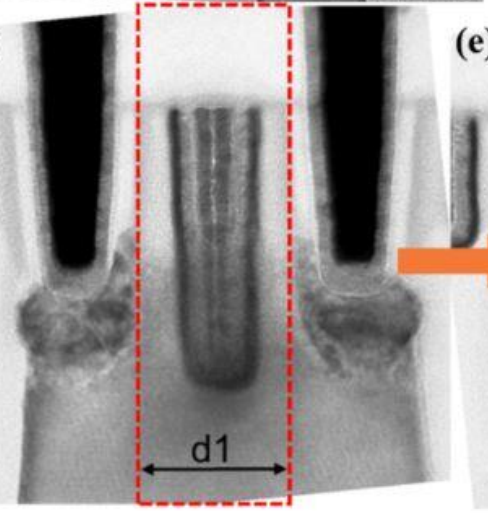

(e)
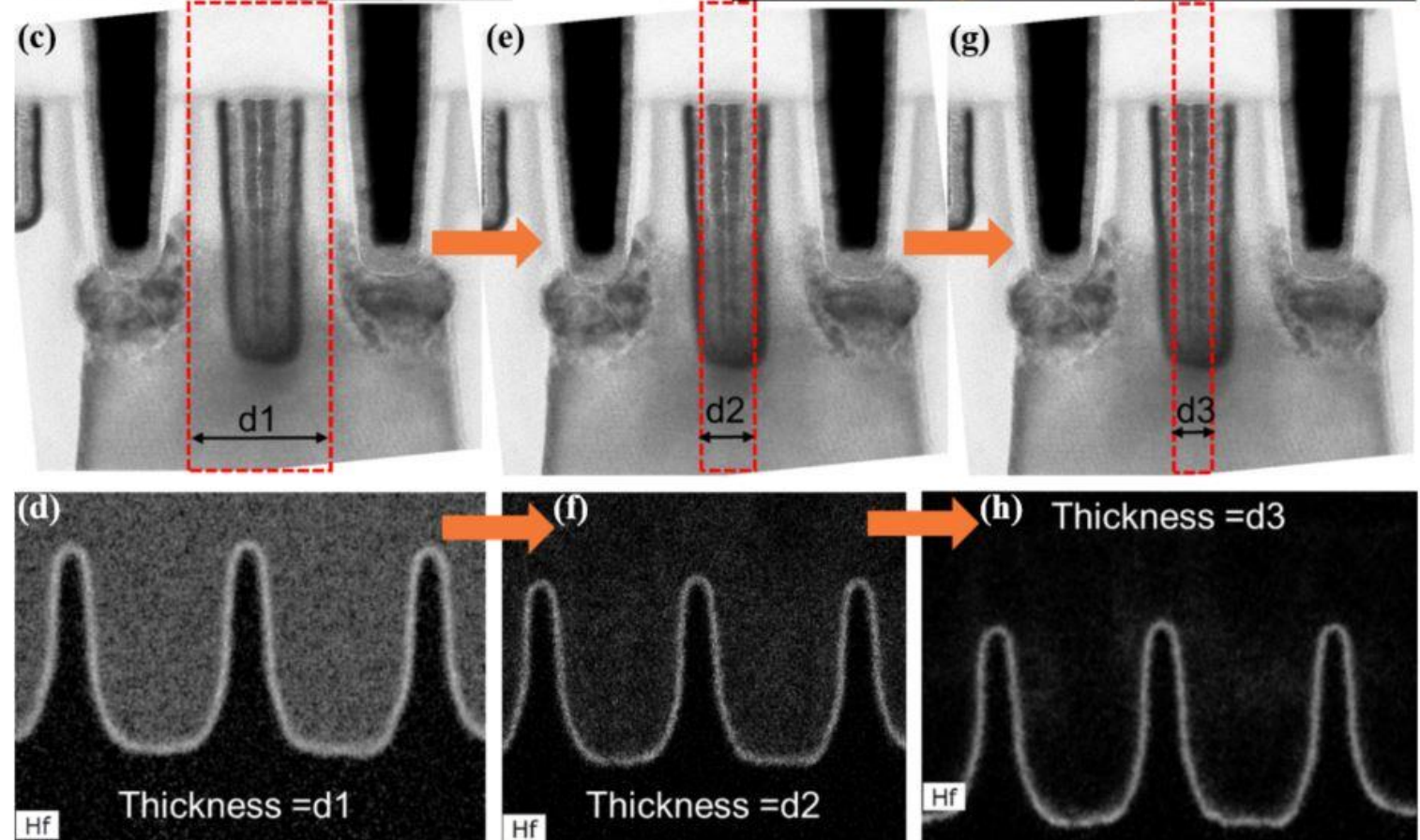

\section{Thickness $=\mathrm{d} 1$}
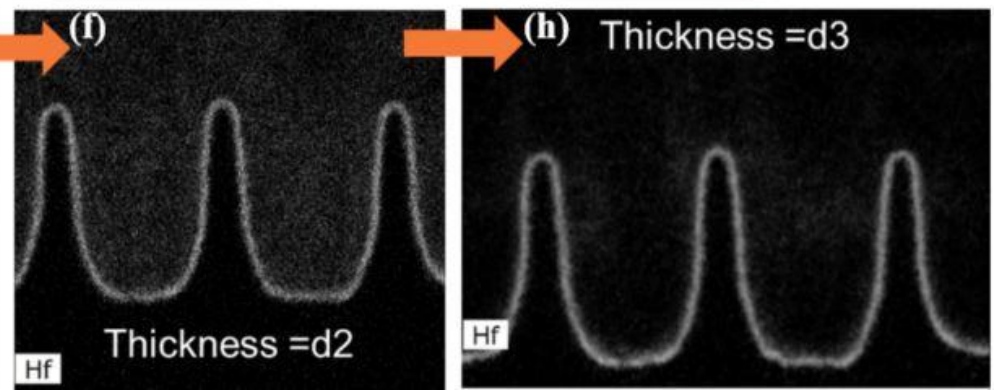

Figure 1. (a)TEM image showing crystal defects at fin tip (highlighted by red arrow and inset) in a thick lamella. It is not clear from the image if the defect resides in the fin or is a projection from the source/drain area; (b)Elemental map showing As and P detected at fin tips (highlighted by red arrows) in a thick TEM lamella. The detected dopant atoms could be located in the channel or in the source/drain area; (c)(e)(g): TEM images illustrating locations of TEM lamella of different thicknesses; (d)(f)(h): Hf maps from TEM lamella of different thicknesses corresponding to (c) (e) and (g). 

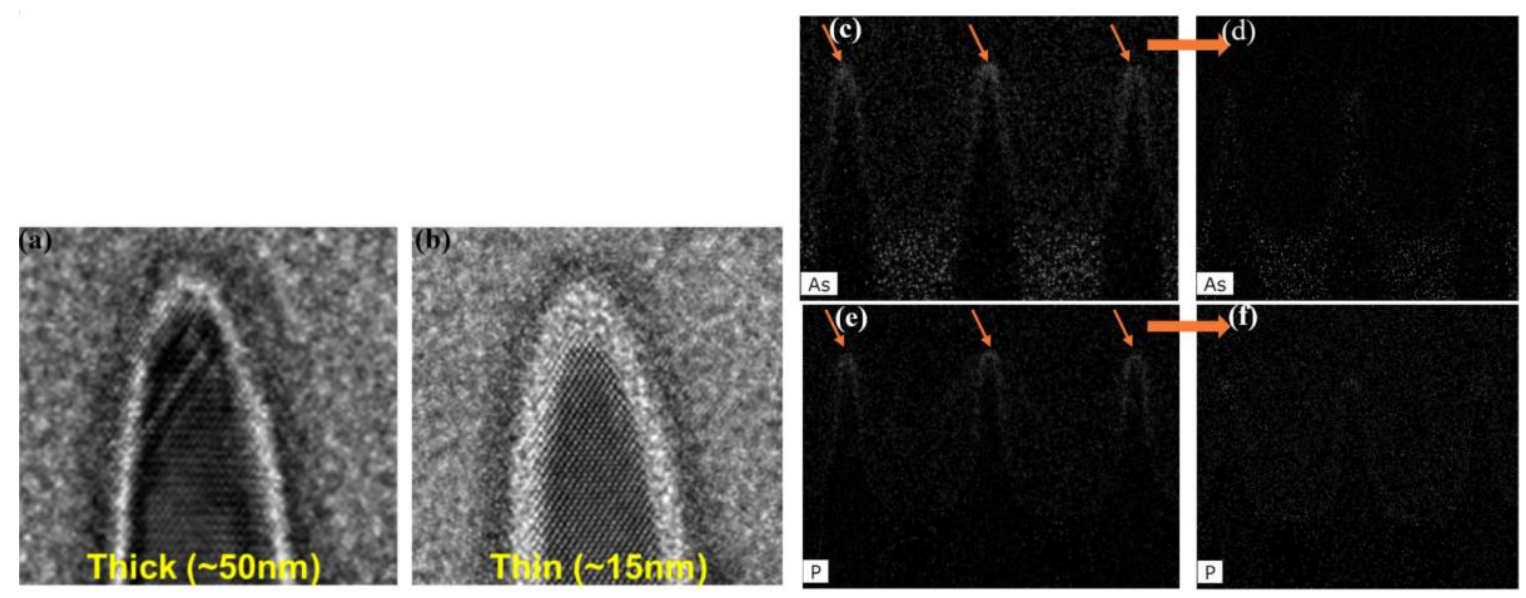

Figure 2. (a)TEM image of fin tip before rethin showing crystal defect; (b)TEM image of same area after rethin with no crystal defect; (c) and (d) Elemental maps of As before (arrowed) and after rethin; (e) and (f) Elemental maps of P before (arrowed) and after rethin.

\section{References}

[1] H. J. Engelmann, H. Saage, E. Zschech, "Application of analytical TEM for failure analysis of semiconductor device structures", Microelectronics Reliability 40, 1747-1751 (2000)

[2] L. A. Giannuzzi, F. A. Stevie, "A review of focused ion beam milling techniques for TEM specimen preparation", Micron 30, 197-204 (1999)

[3] P. H. Albarede, H.J. Lezec, "Transmission electron microscopy of focused ion beam induced damage at $50 \mathrm{keV}$ in Si", Electron Microscopy ICEM14, 431 (1998)

[4] D. Hisamoto, W. Lee, J. Kedzierski, H. Takeuchi, K. Asano, C. Kuo, E. Anderson, T. King, J. Bokor, and C. Hu, "FinFET - A Self-Aligned Double-Gate MOSFET Scalable to 20nm", IEEE Trans. Electron Devices 47, 2320 (2000) 